\title{
Extended Applications of Laser for Photothermal Treatment
}

Seung-Kuk Baek

Department of Otolaryngology-Head and Neck Surgery, Korea University College of Medicine, Seoul, Korea

\section{Correspondence}

Seung-Kuk Baek

Department of Otolaryngology-Head and Neck Surgery, Korea University College of Medicine, Anam-dong 5-ga 126-1, Seongbuk-gu, Seoul 136705 , Korea

Tel: +82-2-920-5486

Fax: +82-2-925-5233

E-mail: mdskbaek@agmail.com

(C) Korean Society for Laser Medicine and Surgery

(c) This is an open access article distributed under the terms of the Creative Commons Attribution NonCommercial License (http://creativecommons.org/ licenses/by-nc/3.0) which permits unrestricted noncommercial use, distribution, and reproduction in any medium, provided the original work is properly cited.

Advances in nanobiotechnology have resulted in newer and improved diagnostic and treatment methods, including ultrasensitive molecular imaging, delivery of chemotherapeutic drugs, and photothermal treatment (PTT). We discuss characteristics and action mechanisms of PTT. Although the safety of nanoparticles has not yet been fully defined, in the future, PTT using nanotechnology may play an important role in treatment of malignant tumor.

\section{Key words}

Photothermal treatment; Nanoparticle 


\section{INTRODUCTION}

Over the past 50 years, although there have been advancements in the management of malignant tumors, the survival rates has shown little improvement and some diseases is still incurable." The cause of unsatisfied improvement in survival may be the limitations of surgical resection due to several vital structures adjacent to tumors, the high failure rate and toxicity of radiation therapy for advanced tumors, poor cell specificity and high toxicity of chemotherapy, and the limited supportive role of chemotherapy in concurrent chemoradiation therapy. Therefore, to overcome the limitation of conventional treatments, the previous studies suggested various alternative treatments, including gene therapy, immunotherapy, and hyperthermia. ${ }^{2-4}$ Among them, since hyperthermia treatment shows synergistic effect with radiation therapy and chemotherapy, hyperthermia treatment, elevating tissue temperature to more than $40^{\circ} \mathrm{C}$, has been used as an adjuvant therapy with chemotherapy and radiotherapy. However, tissue temperature greater than $40^{\circ} \mathrm{C}$ occurs cellular injury or death through protein denaturation and affects both healthy and cancerous tissues. Therefore, the limitation of conventional hyperthermia may be difficulty of targetspecific treatment for malignant tumors.

Recently, photothermal treatment (PTT) using nanoparticles has gained attention as an alternative to hyperthermia treatment through the use of lasers for thermal treatment due to advancements in nanotechnology. The treatment strategy of PTT is similar to photodynamic treatment in terms of the use of light. In photodynamic treatment (PDT), photosensitizer, which absorbs light with specific wavelength, produces reactive singlet oxygen from tissue oxygen and then the reactive oxygen kills tumor cells and cause destruction of tumor vasculature. ${ }^{5}$ On the other hand, three key components of PTT are nanoparticle, light with specific wavelength, and heat. The heat generated by surface plasmon resonance of nanoparticle is used to kill tumor cells.

\section{NANOPARTICLES}

Nanotechnology represents the convergence of multiple scientific fields including chemistry, physics, engineering, molecular biology, and various scientific fields. The advancement of nanotechnology has made nanoparticles to be used to biological field by using various physical properties, including electronic, optical, magnetic, and catalytic characteristics. Especially, the production of nanoparticle with tunable and adequate size and the surface chemical characteristics of nanoparticle, which can be combined various active materials, facilitate extensive application of nanotechnology to biomedical field. ${ }^{6}$ The representative applications of nanoparticle are drug/gene delivery, ${ }^{7-9}$ diagnosis of cancer cells, ${ }^{10-13}$ cancer therapy such as PTT. ${ }^{14-15}$

Nanoparticles can be classified according to composition (gold, carbon, iron oxide, liposomal, dielectric materials), shapes (sphere, rod, star, shell, cage, wire, tube) (Fig. 1). ${ }^{16}$ Among the various nanoparticles currently available, plasmonic gold nanoparticles may be a promising particle because of simple fabrication, multifunctional nature, facile surface chemistry, and relative biocompatibility with low toxicity. ${ }^{16-20}$

\section{SURFACE PLASMON RESONANCE (SPR)}

Metal nanoparticles such as gold nanoparticle and gold nanoshells exhibit unique optical properties that are useful for ultrasensitive detection and photothermal treatment. When the nanoparticles are irradiated by light

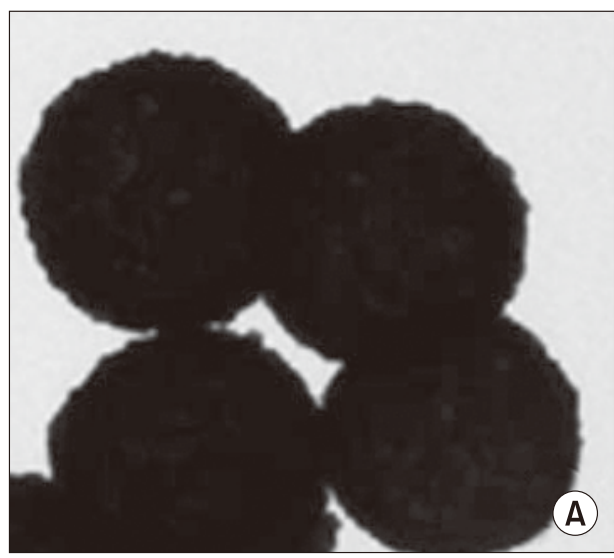

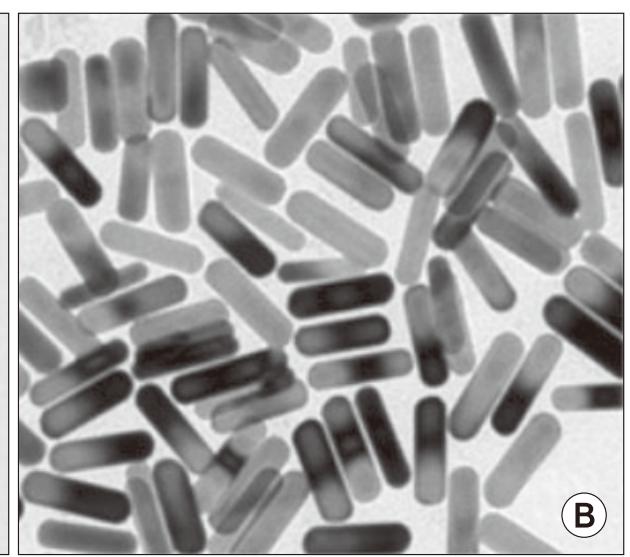

Fig. 1. Nanoshells (A) and nanorods (B). The optical properties of nanoparticle can be modulated according to its' shape, size, and composition. 

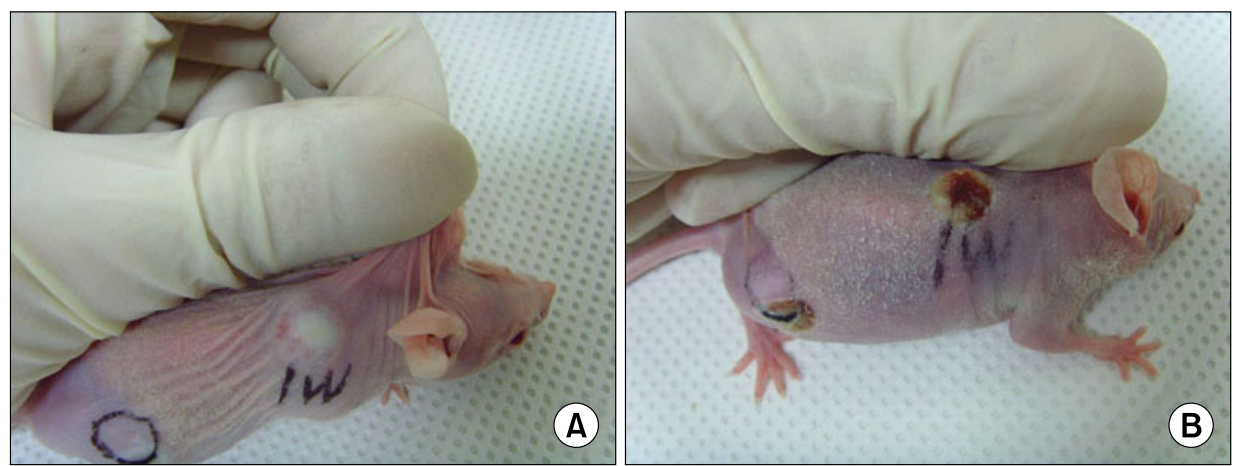

Fig. 2. The nude mouse injected cancer cells subcutaneously. (A) Before photothermal treatment. (B) After photothermal treatment, it shows a partial-destructed tumor mass.

with specific wavelength, the conduction band electrons on the surface of gold nanoparticles oscillate coherently and create a phenomenon known as the SPR. Light energy irradiated on nanoparticles is converted into heat by this phenomenon. Especially, according to the size, shape, composition of nanoparticles, the wavelength of light, in which the particles scatter and absorb light energy, is altered. Therefore, changing the size and shape of gold nanoparticles can alter those peak frequency and they are tunable over the near-infrared region (NIR) of light that has more effective penetration through tissue than the other region of light.

\section{PHOTOTHERMAL TREATMENT (PTT)}

The light absorption by gold nanoparticles can be applied to a generation of heat to kill cancer cells. In addition, the manufacturing of nanoparticle can make the tunable gold nanoparticles to have peak absorption on the near-infrared region of light, where tissue penetration of human tissue by light is maximal. Although the heating temperature around nanoparticles by plasmonic PTT can be increased to $70-80^{\circ} \mathrm{C}$, cellular injury or death results from protein denaturation at temperature greater than $40^{\circ} \mathrm{C}$. Furthermore, since cancerous tissues are more labile to heating than healthy tissues, the effective temperature for PTT effect to kill cancer cells may be from 40 to $50^{\circ} \mathrm{C}$. The target temperature of effective PTT was evaluated in the previous study and we suggested it may be about $45^{\circ} \mathrm{C} .^{21}$ In addition, the cancer cells around nanoparticle-laden macrophages selectively exhibited bubble formation within cytoplasm and cell rupture by irradiating with NIR laser.

In the PTT contrasting with the conventional hyperemia or PDT, which has the limitation of cancer-specificity, the specificity for cancer cells may be achieved by using antibody-conjugated nanoparticles with specificity for surface antigens of cancer or macrophage with phagocytized nanoparticles (Fig. 2).

However, because it is difficult to predict the toxicity of nanoparticles in vivo, PTT using nanoparticles should be performed with a lower concentration of nanoparticles and a lower laser power for a shorter time to prevent injury to the healthy tissue.

\section{CONCLUSIONS}

The advancement of nanotechnology has promoted the clinical application of various types of nanoparticles for diagnosis or treatment of malignant tumor. Even if the toxicity of nanoparticles is an important limitation for clinical application, the various methods increasing specificity for the tumor should enable more selective PTT for cancer cells with reduction of uncontrollable nanotoxicity. If more biocompatible nanomaterials are developed in the future, PTT may be a promising alternative treatment for the malignant tumor.

\section{REFERENCES}

1. Bonner JA, Harari PM, Giralt J, Azarnia N, Shin DM, Cohen $\mathrm{RB}$, et al. Radiotherapy plus cetuximab for squamous-cell carcinoma of the head and neck. N Engl J Med 2006;354:56778.

2. Rosenberg SA. Immunotherapy and gene therapy of cancer. Cancer Res 1991;51:5074s-9.

3. Ambade AV, Joshi GV, Mulherkar R. Effect of suicide gene therapy in combination with immunotherapy on antitumour immune response \& tumour regression in a xenograft mouse model for head \& neck squamous cell carcinoma. Indian J Med Res 2010;132:415-22.

4. Westermann AM, Jones EL, Schem BC, van der Steen-Banasik EM, Koper P, Mella O, et al. First results of triple-modality treatment combining radiotherapy, chemotherapy, and hyperthermia for the treatment of patients with stage IIB, III, and IVA cervical carcinoma. Cancer 2005; 104:763-70. 
5. Castano AP, Mroz P, Hamblin MR. Photodynamic therapy and anti-tumour immunity. Nat Rev Cancer 2006;6:535-45.

6. El-Sayed MA. Some interesting properties of metals confined in time and nanometer space of different shapes. Acc Chem Res 2001;34:257-64.

7. West JL, Halas NJ. Engineered nanomaterials for biophotonics applications: improving sensing, imaging, and therapeutics. Annu Rev Biomed Eng 2003;5:285-92.

8. Paciotti GF, Myer L, Weinreich D, Goia D, Pavel N, McLaughlin RE, et al. Colloidal gold: a novel nanoparticle vector for tumor directed drug delivery. Drug Deliv 2004;11:169-83.

9. Jain KK. Nanotechnology-based drug delivery for cancer. Technol Cancer Res Treat 2005;4:407-16.

10. Wu X, Liu H, Liu J, Haley KN, Treadway JA, Larson JP, et al. Immunofluorescent labeling of cancer marker Her2 and other cellular targets with semiconductor quantum dots. Nat Biotechnol 2003;21:41-6.

11. Chan WC, Maxwell DJ, Gao X, Bailey RE, Han M, Nie S. Luminescent quantum dots for multiplexed biological detection and imaging. Curr Opin Biotechnol 2002;13:40-6.

12. Alivisatos $P$. The use of nanocrystals in biological detection. Nat Biotechnol 2004;22:47-52.

13. Sokolov K, Aaron J, Hsu B, Nida D, Gillenwater A, Follen M, et al. Optical systems for in vivo molecular imaging of cancer. Technol Cancer Res Treat 2003;2:491-504.

14. Hirsch LR, Stafford RJ, Bankson JA, Sershen SR, Rivera B, Price RE, et al. Nanoshell-mediated near-infrared thermal therapy of tumors under magnetic resonance guidance. Proc
Natl Acad Sci U S A 2003;100:13549-54.

15. Baek SK, Makkouk AR, Krasieva T, Sun CH, Madsen SJ, Hirschberg H. Photothermal treatment of glioma; an in vitro study of macrophage-mediated delivery of gold nanoshells. J Neurooncol 2011;104:439-48.

16. Huang X, Jain PK, El-Sayed IH, El-Sayed MA. Gold nanoparticles: interesting optical properties and recent applications in cancer diagnostics and therapy. Nanomedicine (Lond) 2007;2:681-93.

17. Huang X, El-Sayed IH, Qian W, El-Sayed MA. Cancer cell imaging and photothermal therapy in the near-infrared region by using gold nanorods. J Am Chem Soc 2006;128:2115-20.

18. El-Sayed IH, Huang X, El-Sayed MA. Selective laser photothermal therapy of epithelial carcinoma using anti-EGFR antibody conjugated gold nanoparticles. Cancer Lett 2006;239: 129-35.

19. Dickerson EB, Dreaden EC, Huang X, El-Sayed IH, Chu H, Pushpanketh S, et al. Gold nanorod assisted near-infrared plasmonic photothermal therapy (PPTT) of squamous cell carcinoma in mice. Cancer Lett 2008;269:57-66.

20. Niidome T, Yamagata M, Okamoto Y, Akiyama Y, Takahashi H, Kawano T, et al. PEG-modified gold nanorods with a stealth character for in vivo applications. J Control Release 2006;114: 343-7.

21. Yang TD, Choi W, Yoon TH, Lee KJ, Lee JS, Han SH, et al. Realtime phase-contrast imaging of photothermal treatment of head and neck squamous cell carcinoma: an in vitro study of macrophages as a vector for the delivery of gold nanoshells. J Biomed Opt 2012;17:128003. 